How to reference this article Simoncini, F. (2019). Due regine per un'attrice: Maria Stuarda e Elisabetta I nell'interpretazione di Adelaide Ristori. Italica Wratislaviensia, 10(2), 223-237.

DOI: http://dx.doi.org/10.15804/IW.2019.10.1.26

Francesca Simoncini

Università degli Studi di Firenze

francesca.simoncini@unifi.it

ORCID: 0000-0001-6317-5826

\title{
DUE REGINE PER UN'ATTRICE: MARIA STUARDA E ELISABETTA I NELL'INTERPRETAZIONE DI ADELAIDE RISTORI
}

\section{TWO QUEENS FOR AN ACTRESS: MARY STUART AND ELIZABETH I PLAYED BY ADELAIDE RISTORI}

\begin{abstract}
The essay analyses some scenes from Friedrich Schiller's Mary Stuart and from Paolo Giacometti's Queen Elizabeth I of England, performed by Adelaide Ristori. Through a careful comparison between textual sources (memoirs, scripts, testimonies) and iconographic sources (paintings, photographs, engravings), the scene movements and gestures used by the great actress are reconstructed.
\end{abstract}

Keywords: Adelaide Ristori, acting, dramaturgy, Mary Stuart, Elizabeth I 
$\mathrm{N}$ el repertorio di un Grande Attore ottocentesco esistono testi identificabili come "novità", altri come "riprese", altri ancora come "cavalli di battaglia". Ne esistono poi altri, di natura più rara e particolare, che si connotano come indicativi di un intero percorso esistenziale e artistico, anche quando questo è particolarmente lungo e luminoso. Tali copioni entrano in repertorio molto presto quando l'attore è ancora alla ricerca di una affermazione e finiscono col non venire poi mai più abbandonati. Segnano profondamente la formazione di un interprete, ne determinano i punti di svolta, le più significative evoluzioni, i predestinati successi, ne scandiscono i più maturi e gloriosi trionfi. Tra gli attori ottocenteschi e questi copioni si instaura un rapporto così intimo e personale da non concedere possibilità di distacco. Divengono per l'attore o per l'attrice quasi una coazione a ripetere che pretende di raccontare una doppia storia, quella del personaggio protagonista del dramma e, insieme, quella del suo interprete, una storia di vita, di arte e di finzione scenica che cresce e si stratifica nel tempo, varia nelle sue articolazioni, si declina attraverso mille sfaccettature, si fonde con l'immagine stessa dell'attore. Si tratta, in sostanza, di veri e propri testi-biografia.

Il testo-biografia di Adelaide Ristori è Maria Stuarda di Friedrich Schiller. Non a caso è l'opera in assoluto da lei più rappresentata. Chi le ha contate ha computato ben 576 rappresentazioni documentate di questa tragedia, contro le 476 di Elisabetta regina d'Inghilterra di Paolo Giacometti, e le 395 di Medea, solo terza sul podio di questa particolare classifica anche se, forse a torto, considerata dalla storiografia la più rappresentativa della recitazione dell' artista $^{1}$. Ancora più straordinaria è la durata della vitalità di questo copione. Adelaide Ristori incarnò Maria Stuarda a partire dal $1841^{2}$, anno del suo esordio come Prima attrice as-

1 Il computo è stato stilato da Teresa Viziano (2000, p. 50) e si basa su quanto riportato nel manoscritto Repertorio giornaliero delle rappresentazioni 1855-1885 conservato nel Fondo Ristori del Museo Biblioteca dell'Attore di Genova. Al libro di Viziano rimando anche per ulteriori approfondimenti sulla messinscena di Maria Stuarda di Friedrich Schiller (pp. 48-80) e di Elisabetta regina d'Inghilterra di Paolo Giacometti (pp. 162-188).

2 La prima interpretazione di Maria Stuarda di Schiller avvenne a Trento nella primavera del 1841. La traduzione italiana utilizzata da Adelaide Ristori per l'interpretazione della tragedia era quella di Andrea Maffei. 
soluta nella Compagnia Ducale di Parma diretta da Romualdo Mascherpa, fino al suo addio alle scene che volle salutare vestendo ancora, per l'ultima volta, i panni della sfortunata regina scozzese. Accadde a New York il 12 maggio $1885^{3}$. Per ben 44 anni Maria Stuarda e Adelaide Ristori si confrontarono, vissero insieme, si alimentarono l'una dell'altra e interruppero il loro duraturo e straordinario rapporto soltanto con l'abbandono del teatro della Grande Attrice.

L'intenso e esclusivo connubio artistico consumatosi tra Adelaide Ristori e la regina scozzese si allargò, si arricchì di nuovi sensi e di suggestive strategiche risonanze quando, a partire dal 1854, entrò nel repertorio dell'attrice il dramma storico di Paolo Giacometti Elisabetta regina d'Inghilterra. Il nuovo personaggio divenne, con straordinaria e naturale evidenza teatrale, la vivente incarnazione plastica dell'antagonista della sfortunata e prediletta Maria Stuarda. L'ampliamento del repertorio, abilmente costruito grazie a una sapiente drammaturgia d'attrice, permise alla Ristori di esibirsi in un virtuosismo attorico che rasenta l'onnipotenza scenica e che la porta a interpretare, quasi simultaneamente e con sviluppo speculare, sia la vittima sia la carnefice, sia la sconfitta regina martire, sia l'invitta regina vergine: due antitetiche declinazioni di regalità e, insieme, di femminilità ${ }^{4}$. Queste si intrecciano e si rincorrono dando vita a rimandi gestuali e visivi che riescono a costruire corrispondenze e relazioni, fino a creare un forte e magnetico bipolarismo scenico che, anche se recitato in modo differito, si avvale di una stretta contiguità di rappresentazione. Le trame dei due testi, recitati entro un ristretto arco temporale, permettono all'attrice di costruire un ideale montaggio narrativo che la Ristori sembra suggerire con forza agli spettatori, chiamati a ricucire autonomamente le scene "scannate" di una storia per sua natura unitaria, anche se rappresentata in tempi sfalsati e da opposti punti di vista.

Ben scolpite fin dall'inizio appaiono le linee interpretative dei due personaggi. Maria Stuarda incarna l'immagine di una perfetta regina-

\footnotetext{
3 Nell'occasione Adelaide Ristori recitò il dramma con una compagnia tedesca.

4 Nelle sue tournées la capocomica faceva seguire, a distanza di pochissimi giorni l'una dall'altra, la recita di Elisabetta regina d'Inghilterra a quella di Maria Stuarda.
} 
martire, nobile nell'indole, vilipesa nella dignità, sofferente per la prolungata e ingiusta prigionia, rassegnata nell'accettazione del suo destino e tuttavia non piegata dagli eventi e ancora pienamente in possesso di una energica e aristocratica vitalità. Tratti pertinenti di un ritratto potente, contrastato e dinamico che dovevano essere colti immediatamente dal pubblico e suggeriti a partire dal costume indossato che fu, e rimase, unico per tutta la durata della tragedia. Troppo importante dunque per lasciare che fossero soltanto le indicazioni del testo a disegnarne la preziosa tessitura. Per concepirlo e perfezionarlo la Ristori si ispirò ai ritratti dell'epoca rifacendosi in particolare a un quadro, da lei citato nelle sue Memorie, dove la Stuarda è raffigurata in piedi e: «indossa un abito di velluto nero impresso sormontato da una zimarra senza maniche. Una randiglia bianca le sormonta il collo, in capo porta una cuffia di trina bianca (...) e la copre interamente fino a terra il velo parimenti bianco» (Ristori, 2005, pp. 133-134) . Sostituito il colore nero al bianco della cuffia e del velo, la Ristori dichiara di aver indossato per vestire il personaggio esattamente lo stesso abito, adornato solo da un crocifisso dorato, evidente sostituto della corona negata. Un oggetto di intensa pregnanza simbolica che, impugnato dalla Ristori, acquistava vitale dinamismo scenico. Ulteriori riscontri con la fonte iconografica su cui l'attrice modellò la sua interpretazione restituiscono l'impronta visiva che, fin dalla prima apparizione, l'attrice volle imprimere sul pubblico: Adelaide Ristori intese incarnare una regina prigioniera e sofferente, ma ancora giovane, bella, alta e flessuosa, eretta nella persona, serafica e sorridente.

Ben cinque, uno per ogni atto, furono invece $\mathrm{i}$ costumi indossati per l'interpretazione di Elisabetta I, concepiti quali sfarzose sottolineature del ritratto di una donna volubile, capricciosa, ipocrita e dissimulatrice, a tratti irascibile, ma comunque dotata di regale dignità e di non comune ingegno: «tipo unico, piuttosto che raro, di donna e di sovrana» (Ibidem, p. 141) ${ }^{6}$ la definisce nel suo studio artistico l'attrice-marchesa.

\footnotetext{
5 Prima edizione del testo: 1887, Torino-Napoli: L. Roux e C. Editori.

6 Per un'ampia descrizione dei costumi indossati dalla Ristori per impersonare Elisabetta I si veda Viziano, 2000, pp. 171-188.
} 
Solo nel copione di Schiller le due regine si incontrano. Avviene nella scena centrale del dramma, la terza del terzo atto. Consapevole che il nucleo drammaturgico dell'opera era racchiuso in questa scena, Adelaide Ristori si adoperò per renderla memorabile e la scolpì facendo ricorso a una sequenza di gesti ben calibrati che, ancor più che impressivi, dovettero risultare sconcertanti e impressionanti per gli spettatori, tali da fissarsi profondamente e indelebilmente nella loro mente. Fu in questo punto della tragedia che la recitazione di Adelaide Ristori raggiunse il suo culmine toccando l'apice di un virtuosismo scenico di rara e sapiente qualità che si segnala per la capacità di risolvere, armonizzandoli con compostezza, i contrastanti stati d'animo del personaggio. Fu una scena in cui la regale e rassegnata dignità apollinea della regina martire, fino ad allora prevalentemente recitata dalla Ristori, riuscì a conciliarsi artisticamente con l'improvviso scatenarsi di un vitale furore dionisiaco, latente, ma comunque presente, nell'indole della regina impersonata dall'attrice.

Alcune fotografie, una caricatura, lo studio della parte che l'attrice consegnò alle stampe, una testimonianza oculare, permettono di ricostruire le principali sequenze di questa scena. L'analisi comparata delle fonti, diverse per tipologia e provenienza, restituisce, con accettabile approssimazione, la partitura mimica e gestuale con cui Adelaide Ristori risolse il difficile contrasto.

La scena - come detto - pone a confronto Maria Stuarda con Elisabetta I d'Inghilterra, usurpatrice del suo regno e sua spietata carceriera. Il pathos drammaturgico è qui al culmine, carico di una tensione che aggiunge agli eventi passati - stratificatisi in diciannove anni di prigionia - quelli, sul momento drammaticamente presenti e incalzanti: la condanna a morte di Maria Stuarda è già stata decretata e attende soltanto l'approvazione di Elisabetta per essere portata ad esecuzione. I pochi fedelissimi che ancora la circondano esortano la Stuarda a chiedere un incontro riconciliatore con la regina d'Inghilterra per ottenere da questa la sospirata grazia. Il dialogo tra le due rivali presuppone dunque l'adozione, da parte della protagonista, di un comportamento di umile e compunta sottomissione, certamente consono all'immagine della regina martire, ma di fatto inconciliabile con quello della altera e fiera sovra- 
na, pur avvezza ormai da tempo a vestire i panni della fervente penitente fino ad identificarsi intimamente con i valori, mistici e spirituali, della fede cattolica.

I due poli fondativi dell'identità scenica di Maria Stuarda, che pretendono l'evidenza di una dignitosa e altera regalità come quelli dell'umiltà spirituale della martire, sono teatralmente funzionanti e praticabili se collocati in azioni sceniche che ne prevedono una separata collocazione, ma rischiano di crollare miseramente nella loro resa interpretativa se costretti a coesistere e a entrare in conflitto tra loro. Lungi dal sottrarsi dal recitare questo impasse Adelaide Ristori spinse sull'acceleratore della inconciliabilità tra i due opposti sentimenti e mostrò, con chiara e plastica evidenza, l'inesorabile disgregarsi della identità di Maria Stuarda. Qualcosa improvvisamente e vistosamente cominciò a incepparsi nella fluidità dei suoi movimenti e nell'articolazione della sua, di solito perfetta, dizione. L'immagine della rassegnata penitente, lentamente, per gradi, pezzo dopo pezzo, si scompose sotto gli occhi degli sbigottiti spettatori, ormai abituati ad assumerne i tratti nobili e composti. Come in una danza bacchica, costruita attraverso bruschi scarti e arresti repentini, l'icona della regina martire scompare e ricompare nei gesti dell'attrice permanendo in scena ad intervalli sempre più brevi e concedendo via via sempre più spazio all'inesorabile insorgere di una gestualità di segno contrario, improntata a sottolineare l'incontrastabile sopravanzare di un'ira a stento repressa e, di fatto, incontenibile.

A nulla serve aggrapparsi agli strumenti della fede, quegli oggetti di scena, il rosario e il crocifisso, finora indossati come semplici elementi decorativi dell'abito e che adesso, invece, improvvisamente, si animano, acquistando significato e dinamismo scenico. Ristori-Stuarda stringe convulsamente il rosario, cerca disperatamente di ancorare la sua residua remissività al crocifisso che tiene stretto in mano e che porta febbrilmente al petto invocando l'aiuto divino: l'ira, selvaggia e furiosa, aizzata dalle arroganti parole della rivale, alla fine prorompe. Ed è, come si conviene, potente e terribile.

La violenta intensità del movimento scenico creato dall'attrice è documentata da una caricatura (Fig. 1) e dalla testimonianza di un atten- 


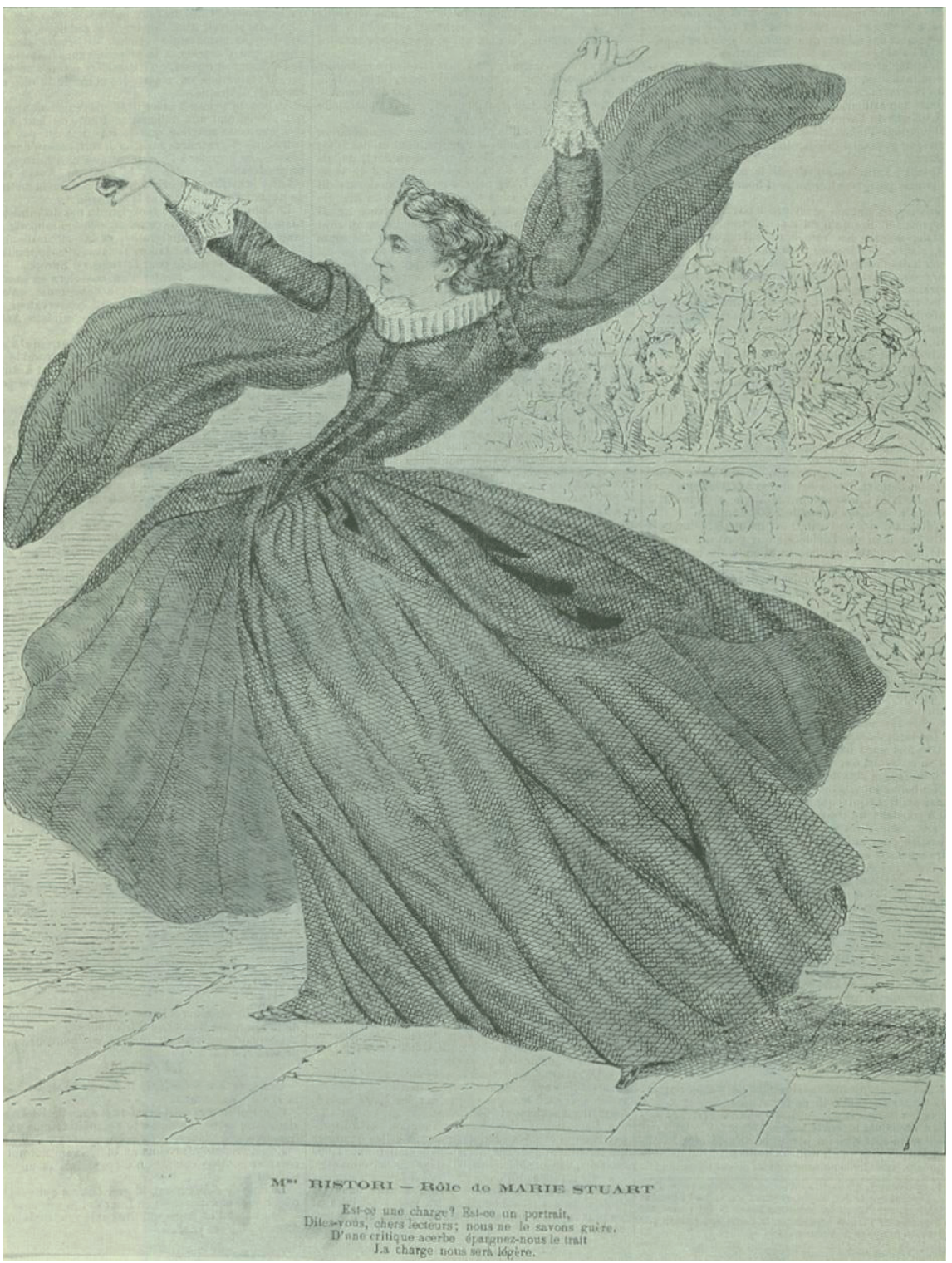

FIg. 1. Caricatura di Adelaide Ristori in Maria Stuarda. La foto è tratta da un ritaglio di stampa di un giornale francese privo di indicazioni e è pubblicata in Viziano, 2000, p. 64. 
to spettatore, August Bournonville ${ }^{7}$, che vide Adelaide Ristori recitare a Vienna intorno alla metà degli anni Cinquanta. Il testimone fissò su carta la descrizione di quella particolare, sconcertante, metamorfosi che l'attrice seppe mostrare in scena nel suo divenire:

In ginocchio di fronte alla regina d'Inghilterra, ella stringe convulsamente la sua corona del rosario, come se volesse significare che non è di fronte a una potenza terrestre che in quel momento si umilia. Un brivido l'attraversa, allorché il suo sguardo incontra il volto glaciale della sua rivale; cerca con ardente eloquenza di piegare il duro animo di quella donna altezzosa - ma invano! Contrastando le sue indignate emozioni, invoca dal cielo la forza e la pazienza di sopportare le offese, con le quali l'intransigente persiste a investirla; ma alla fine non ce la fa più: vuol rispondere, ma la voce le viene meno, e solo dopo una violenta lotta spirituale vengono fuori le parole. Come una furiosa tempesta ora sono scagliati i dardi velenosi dell'odio e della vendetta su "la figlia di Anna Bolena". Maria la sbatte quasi fuori scena, la chiama "mima bastarda" e invoca la giustizia divina, per vedere Elisabetta prostrata nella polvere e se stessa sul trono d'Inghilterra. (Perrelli, 2004, p. 18)

La forza dell'indemoniato furore dovette durare soltanto pochi attimi ma tali da rivelare non solo un personaggio del tutto diverso rispetto a quello, sapientemente e lungamente tratteggiato dall'attrice fin dal primo atto, ma anche l'adozione di un diverso e anticonvenzionale stile recitativo. Alle pose accademiche, statuarie e quasi ieratiche, che erano il vanto internazionale della recitazione di Adelaide Ristori e che fino ad allora avevano caratterizzato l'incarnazione della regina-martire, si sostituirono l'atletica energia acrobatica e le qualità ginniche proprie della figlia d'arte, appartenenti alla bassa tradizione attorica italiana.

7 August Bournonville (1805-1879), importante personalità del teatro danese ottocentesco, dedica alcune pagine della sua autobiografia Mit Teaterliv. Erindringer og Tidsbilleder (København, Thaning \& Appel, 1979) alla descrizione delle recite date da Adelaide Ristori a Vienna intorno agli anni Cinquanta del secolo. Traggo la citazione da Perrelli, 2004. I corsivi sono nel testo. Al libro di Franco Perrelli rimando per una approfondita panoramica delle impressioni che gli spettatori scandinavi ricevettero dell'arte di Adelaide Ristori osservata durante le tournées scandinave degli anni 1879 e 1880 . 
Ecco allora che Maria Stuarda, così completamente ridisegnata dall'attrice, incalza fisicamente Elisabetta, la aggredisce spingendola violentemente fuori scena non diversamente da come farebbe uno schermitore con il suo avversario in pedana. Appena però ottenuto il successo e affermata la propria supremazia il personaggio immediatamente si ricompone riappropriandosi di quella nobile e fissa dignità che fin dall' $\mathrm{i}-$ nizio l'aveva connotato. Il movimento indiavolato si arresta con una sospensione che dura qualche istante e che fornisce all'attrice il tempo sufficiente per recuperare il gesto e la posa scultorei con cui, anche quella scena, avrebbe dovuto chiudersi, in bellezza e compostezza (Fig. 2). Lo certifica, ancora, il nostro prezioso testimone:

L'azione si bloccava diversi minuti, durante i quali Maria, sopraffatta dall'emozione, restava fremente, con le labbra esangui e gli occhi fiammeggianti, indicando col dito fuori verso la regina fuggente, come a voler dire: "Colpita!" (Ibidem)

Nella pièce di Giacometti le vicende relative alla Stuarda, che pur costituiscono una parte importante dell'azione, sono invece raccontate in assenza della rivale e vissute, quasi esclusivamente, nell'intimità turbata di Elisabetta a cui, dopo l'avvenuta decapitazione, appare più volte lo "spettro" della cugina uccisa. Memorabile, nel ricordo dei testimoni, è l'interpretazione del momento in cui la regina d'Inghilterra è chiamata a firmare la condanna a morte di Maria Stuarda. La scena, ideale prosecuzione narrativa di quella recitata nel copione di Schiller - da noi appena raccontata - permette alla Ristori di prodursi in una prova interpretativa giocata sull'uso sapiente delle controscene, chiamate ad evidenziare uno dei tratti pertinenti del personaggio: l'ipocrisia.

Quando il guardasigilli, sir Darwiston, nella scena quarta del secondo atto del dramma, reca alla regina l'ultima lettera della rivale e, insieme a questa, la sentenza della condanna a morte, l'attrice «a stento reprime un'espressione di gioia, ed ipocritamente copre la sua emozione con la larva della pietà» (Ristori, 2005, p. 145). Il gioco scenico della simulazione si protrae nelle azioni successive che mostrano la sovrana fingere un dolore marcatamente esibito solo per mascherare l'intima soddisfazione provata per l'annientamento della regina scozzese, le- 


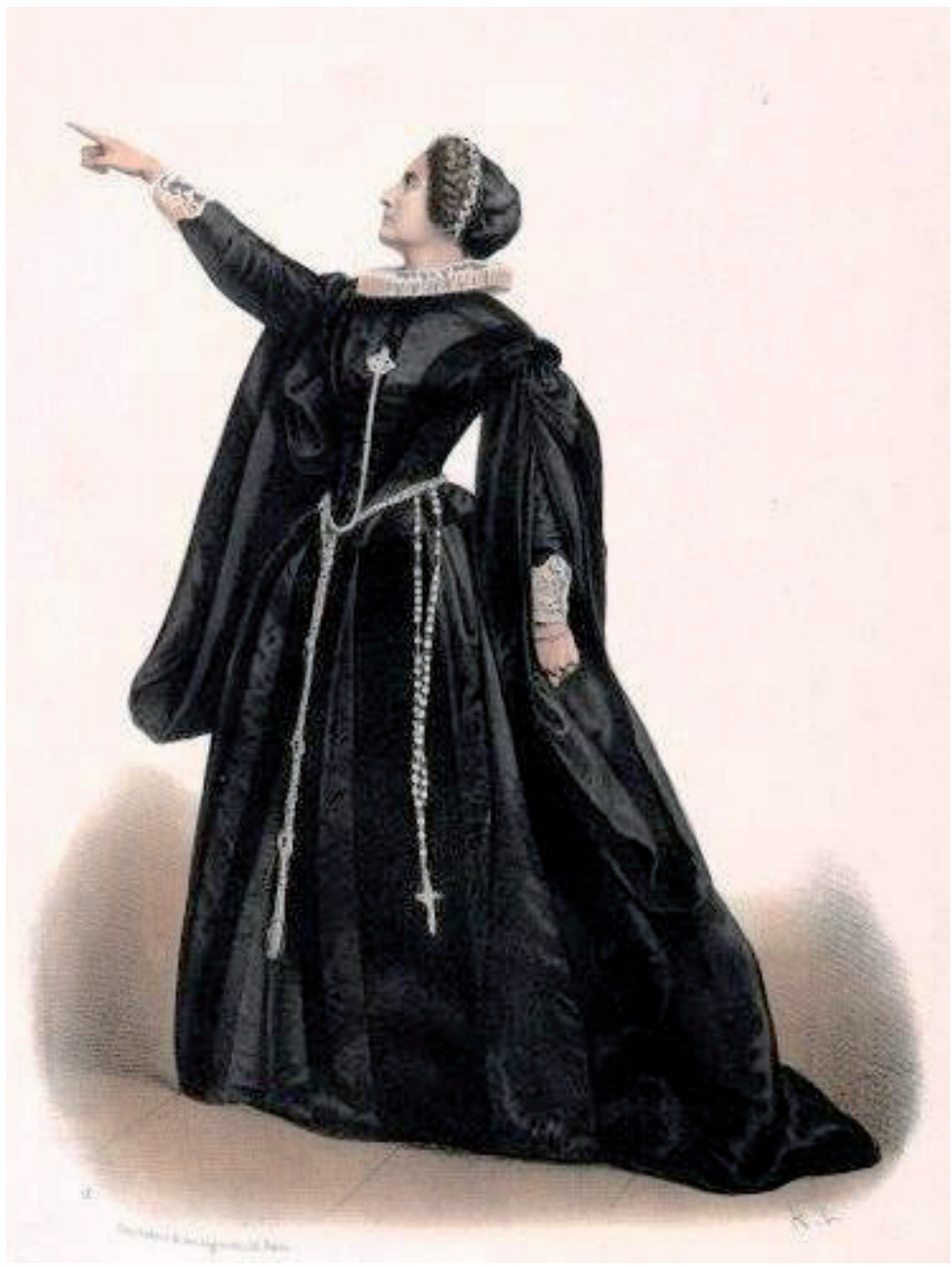

Fig. 2. Adelaide Ristori in Maria Stuarda. Stampa. Album Artistique,

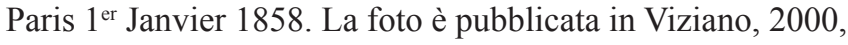
p. 62 .

gittima aspirante al trono di Inghilterra. All'annuncio della morte della Stuarda, racconta l'attrice nelle sue memorie:

$[\ldots]$ in un baleno tutta la mia persona subiva una completa trasformazione, ed un grido di gioia mi sfuggiva dal petto, grido che nella costernazione per il terribile annunzio andava inosservato, e che tosto io reprimevo irrompen- 
do con furore contro coloro che avevano fatto eseguire la sentenza. Con la stessa rapidità improntavo instantaneamente sul mio volto tale espressione esagerata di dolore, tali smanie insensate, da trarre in inganno perfino lo stesso Giacomo VI. (Ibidem, pp. 145-146)

Chi la vide confermò i repentini scarti di tono eseguiti, con abile virtuosismo scenico e consumata arte teatrale dalla Grande Attrice. Così si espresse il cronista del "Dagens Nyether" che, il 21 ottobre del 1879, assistette all'interpretazione e la definì:

$[\ldots]$ un capolavoro di recitazione facciale $[\ldots]$ si trema e si gela ogniqualvolta si vede il suo occulto scherno mutare nell'espressione della più profonda emozione, mentre promette a Giacomo di graziarne la madre. E in seguito quale perfetta mimica quando, dopo il delitto, si accascia per l'afflizione e quindi con parole di condanna fulmina il povero Davison e infine semicontrita per la devozione dichiara il suo proposito di ritirarsi in convento! (Perrelli, 2004, p. 40)

Proprio verità, da una parte, e ipocrisia, dall'altra, costituiscono i due poli chiamati a connotare la diversa indole delle due regine rivali come esplicitato nella furiosa invettiva che, nel terzo atto del dramma di Schiller, la Stuarda lancia contro Elisabetta. Queste le parole usate nel copione di Adelaide Ristori conservato presso il Fondo Ristori del Museo Biblioteca dell'Attore di Genova:

Umano / Fu l'errore che mi vinse in giovinezza: / Mi tradì la potenza: Io no '1 copersi, / Io no ' 1 mentii, Con nobile alterigia /Sdegnai la tenebrosa arte dei vili. / Il peggio è di me noto, e dir mi posso / Di mia fama miglior. Te sciagurata; / Se cade un giorno l'onorata veste / Di cui ricopri, ipocrita maligna, / L'oscena tresca dei tuoi sozzi amori! ${ }^{8}$

Molte ancora sarebbero le scene dei due drammi da analizzare in modo sinottico per dare evidenza critica a un discorso fin qui, per

8 Presso il Museo Biblioteca dell'Attore di Genova sono conservati alcuni copioni e parti levate del testo di Schiller, tra questi un copione manoscritto con visti di censura da cui traggo la citazione. Secondo Teresa Viziano (2000, p. 48, nota 1), è questo il copione che Adelaide Ristori usò nella famosa tournée parigina del 1855. 
necessità, appena abbozzato. Molteplici inoltre le fonti da interrogare (copioni, recensioni, fotografie, bozzetti), per riuscire a comprendere l'originale lavoro condotto dall'attrice su entrambi i testi. Per ovvie ragioni di spazio, scegliamo di fornire qui soltanto un ulteriore unico, ma significativo, esempio comparando le scene finali delle due pièces in cui, entrambe le eroine, vanno fatalmente incontro alla morte.

Davanti all'inesorabilità del suo destino Maria Stuarda esibisce una dolce, suadente, pacata femminilità, a tratti fragile, a tratti energica e dignitosa, sublimata, sempre, da una profonda e fervente religiosità. La tranquilla rassegnazione con cui la regina si avvia alla morte rivela la parabola di una esistenza condotta senza dissimulazioni e infingimenti, pienamente vissuta dalla Stuarda, sia quando, in età giovanile, erano stati gli amori scomposti e la gloria e l'ambizione a prevalere, sia quando, nei diciannove anni di prigionia, aveva invece preso il sopravvento il sentimento sincero di una spiritualità intensa e appagante. All'opposto la morte di Elisabetta si configura come una straordinaria scena di follia, mirabilmente concepita e interpretata. La Ristori riesce a trasformare l'ultimo atto del dramma di Paolo Giacometti, dai più giudicato mediocre, in un alto momento di virtuosismo recitativo. I tratti sublimi, raggiunti dalla Stuarda negli attimi che precedono la sua esecuzione, non si addicono ad una regina che aveva negato a se stessa la vita d'amore e inibito all'anima i più puri sentimenti muliebri sacrificandoli all'altare del regno e del potere. La recitazione della Ristori assume allora, nell'interpretazione di Elisabetta morente, venature di crudo e inconsueto realismo mentre $i$ comportamenti della regina, che alterna a momenti di residua regalità momenti di sconcertante perdita di lucidità e di controllo, si ammantano di venature grottesche.

Ormai vecchia ${ }^{9}$, provata dal male, logorata dai rimorsi e tormentata dai fantasmi di coloro che aveva fatto giustiziare - Maria Stuarda e l'amato conte di Essex - la regina, spaventata, si aggrappa nervosa-

9 Così Adelaide Ristori descrive il suo ingresso in scena nel V atto: «Nell'apparire sulla scena, il mio aspetto presentava le alterazioni che l'età avanzata doveva avere operato sul mio volto, nonché l'impronta del malore che lentamente mi consumava [...]. La trascurata acconciatura dei capelli, le rughe del volto, il tardo movimento delle braccia, delle mani, del passo, dovevano far indovinare al pubblico come più 
mente a una corona che le è divenuta pesante e che non riesce più a portare. In questa scena del dramma, racconta Adelaide Ristori:

Il ricordo della morte d'Essex, da me stessa ordinata, mi dilania il cuore; i rimorsi mi straziano, mi prostrano, provo il bisogno di gettarmi sul letto. Io mi vi trascinavo a stento, con il corpo piegato, il capo chino, e ponendomi le mani alla fronte, toccavo la corona che la cingeva. "Ah! essa è pure un gran peso", dicevo sospirando, "eppure l'ho portata per 44 anni e mi è sembrata sì lieve!" (2005, p. 154)

È questa la premessa del progressivo sfaldarsi dell'integrità del personaggio che, gradualmente e con intermittenze, precipita inesorabilmente verso la follia:

Il respiro cominciava a farsi affannoso, l'occhio ad offuscarsi, la mente a sconvolgersi. [...] Mi figuravo vedermi avvolta dalle tenebre, scorgeva ombre bianche o spettri insanguinati venirmi incontro. Per sfuggirli e non essere afferrata da loro, mi rannicchiava nel letto; ma i capi recisi, che mi sembravano rotolare ai miei piedi, mi atterrivano, ed in preda a spasimi orrendi cadevo nuovamente sul letto, chiedendo a mani giunte, e con voce soffocata, misericordia e pietà! (Ibidem, p. 155)

La vista di Giacomo, figlio di Maria Stuarda e suo designato successore, provoca un'ulteriore scossa nella mente già sconvolta della regina. Le sembra che rechi in mano la testa mozzata della madre. Ne è atterrita. Gridando si rifugia tra le braccia dei suoi più fedeli consiglieri e si copre il volto con entrambe le mani «come per respingere il contatto del mozzo capo della Stuarda» (Ibidem). Quindi si placa recuperando barlumi di lucidità.

Lo sterile esercizio del potere ha però reso asessuata l'invincibile regina disumanizzandola e facendola apparire agli spettatori più simile ad un animale ferito che a un essere umano. Con furia selvaggia e con un impressionante e feroce balzo ferino questa donna, ormai morente e fisicamente informe, strappa violentemente la corona dal capo di Gia-

dell'età, un dolore che gelosamente celavo nel cuore logorasse la mia esistenza» (2005, pp. 152-153). 
como I, figlio di Maria Stuarda, un attimo prima da lei stessa incoronato re d'Inghilterra. Un gesto impressionante e potente ma, insieme, risibilmente patetico a cui la Ristori fa immediatamente seguire l'inesorabile delirio dell'agonia.

È con tali antitetici accenti che le due donne, insieme rivali e regine, terminano la loro esistenza, l'una raggiungendo il sublime, l'altra precipitando in un realismo grottesco che sfiora il tragicomico. Allo spettacolo della loro fine sopravvive, trionfante, l'attrice-marchesa, più di loro capace di conciliare, con armoniosa sintonia, nobiltà e maternità, amore coniugale e indefesso lavoro, finzione d'arte e realtà di vita. Un esempio di inimitabile e irraggiungibile perfezione che certo non sfuggiva a chi la vedeva incarnare sul palco regine di lei più famose, ma a loro modo imperfette. Le platee di tutto il mondo la applaudivano certo anche per questo, per quella meravigliosa e ideale creazione che Adelaide Ristori aveva saputo fare di sé e della sua vita e che mai, finché visse, smise di recitare.

\section{BIBLIOGRAFIA}

Brunelli, B., \& Pastina, G. (1961). Ristori, Adelaide. In Enciclopedia dello spettacolo (vol. 8, coll. 1015-1018), Roma: Le Maschere.

Lenzi, M. (1993). L'attore iperboreo. Le figurazioni sceniche di Adelaide Ristori e Ernesto Rossi nel prisma della critica russa contemporanea (1860-1896). Pisa: ETS.

Leonelli, N. (1944). Attori tragici attori comici, vol. 2. Roma: Tosi.

Mariani, L. (2017). L'ottocento delle attrici. Acting Archives Review, 7(14), 1-26. Retrieved from https://www.actingarchives.it/review/archivionumeri/24-anno-vii-numero-14-novembre-2017/155-1-ottocento-delleattrici.html.

Pandolfi, V. (1954). Antologia del grande attore. Bari: Laterza.

Perrelli, F. (2004). Echi nordici di grandi attori italiani. Firenze: Casa Editrice Le Lettere.

Rasi, L. (1905). I comici italiani, vol. 3. Firenze: Fratelli Bocca.

Regli, F. (1860). Dizionario biografico dei più celebri poeti ed artisti melodrammatici, tragici e comici, maestri, concertisti, coreografi, mimi, bal- 
lerini, scenografi, giornalisti, impresarii, ecc., che fiorirono in Italia dal 1800 al 1860. (pp. 448-450). Torino: Enrico Dalmazzo.

Ristori, A. (1887). Ricordi e studi artistici. Torino-Napoli: L. Roux e C. Editori. Ristampa: a cura di A. Valoroso (2005). Roma: Dino Audino.

Tinterri, A. (2016). Ristori, Adelaide. In Dizionario Biografico degli Italiani, vol. 87. Roma: Istituto dell'Enciclopedia Italiana. Retrieved from http:// www.treccani.it/enciclopedia/adelaide-ristori_(Dizionario-Biografico).

Viziano, T., \& Tinterri, A. (Eds.). (1978). La monarchia teatrale di Adelaide Ristori. Firenze: Mori.

Viziano, T. (Ed.). (1982). Paolo Giacometti, Adelaide Ristori: diritto d'autore, diritto d'attrice. Novi Ligure: Arti grafiche novaresi.

Viziano, T. (2000). Il palcoscenico di Adelaide Ristori. Repertorio, scenari e costumi di una Compagnia Drammatica dell'Ottocento. Roma: Bulzoni.

Viziano, T. (2013). La Ristori. Vita romanzesca di una primadonna dell'Ottocento. San Miniato (PI): La conchiglia di Santiago.

Riassunto: Il saggio analizza alcune scene di Maria Stuarda di Friedrich Schiller e di Elisabetta I regina d'Inghilterra di Paolo Giacometti interpretate da Adelaide Ristori. Attraverso un'attenta comparazione tra fonti testuali (memorie, copioni, testimonianze) e fonti iconografiche (quadri, fotografie, incisioni) vengono ricostruiti i movimenti di scena e la gestualità usati dalla Grande Attrice per delineare e rendere scenicamente i caratteri e le psicologie delle due regine rivali.

Parole chiave: Adelaide Ristori, recitazione, drammaturgia, Maria Stuarda, Elisabetta I 